\title{
A hybrid Model for the Evolution of Network Public Sentiment Based on Bass and Cellular Automata
}

\author{
Shibao Sun ${ }^{\mathrm{a}}$, Jingshan Zhang ${ }^{\mathrm{b}}$, Yanan Zhang $^{\mathrm{c}}$ and Chong Zhang ${ }^{\mathrm{d}}$ \\ College of Information Engineering, Henan University of Science and Technology, Luoyang 471023, \\ Henan, China \\ àsunshibao@haust.edu.cn, bsuperzhangjs@126.com, 'zyn_haust@126.com, dzhangchong1970@1 \\ 63.com
}

Keywords: Network Public Opinion; Cellular Automata; External Influence; Simulation Experiment.

\begin{abstract}
Considering netizen opinion is influenced by many factors in the spread of public opinion, combine the Bass theory and traditional cellular automata model, add external influence, degree of neighbor and external influence, a hybrid model for the evolution of online public sentiment considering the external influence is set up. Propose a modified conversion formula of tendency, analyze simulation results by coarse and fine grained statistical method. The simulation results show that the model can better reflect the trend of network public opinion dissemination.
\end{abstract}

\section{Introduction}

With the development of the Internet, network public opinion as an important part of social public opinion is playing an increasingly important role, the study of network public opinion in China began in 2005. Definition of network public opinion by Liu Yi is: the sum of the various emotions, attitudes and opinions expressed and transmitted through the Internet. Network public opinion has the characteristics of freedom, interactivity, diversity, deviation and sudden, so it is very difficult to study directly.

Cellular automaton is a kind of mathematical model space and time are discrete, which is suitable for studying the nonlinear change of complex systems, many scholars at home and abroad use it for the study of public opinion dissemination. Alves [1] proposed that the microscopic discrete dynamics model based on local interaction will gradually replace the macro model of public opinion, and introduced the cellular automaton model for the first time in public opinion research. Zeng Xiankui [2] first proposed collaborative cellular automaton. Liu Muren [3] and Fang Wei [4] studied the cellular automata based on the global and local majority rules, Fang Wei focused on the influence of the main cell firmness and neighborhood on the opinion propagation. Zeng Xiangping [5] considered the influence of individual neighbors' views on the subject tendency, represents the main point of the view affected by the degree of confidence. Wang Gensheng [6] put forward a migrating cellular automata model, study the evolution of public opinion by mean method.

The current cellular automata simulation models are based on the local rules of the evolution of network public opinion, only consider the influence of neighborhood on the subject, but people's opinions in real life are not only influenced by the tendency of individuals around, but also by the overall trend of public opinion, news media and government guidance and other factors. In order to break through the limitations of the traditional research, in this paper, a hybrid cellular automaton model is proposed by combining the Bass model with cellular automata to simulate the propagation process of public opinion in reality, make public opinion research more reliable.

\section{Cellular automata model based on Bass}

Cellular automata(CA) is a kinetic model of discrete time and space, is suitable for studying temporal dynamic evolution of complex systems. CA is composed of discrete cellular, each cell takes a finite discrete value and updates synchronously according to certain conversion rules.Cellular automata can be represented as a four tuple: $C A=\left(A^{N}, \Sigma, \mathrm{E}, \mathrm{F}\right), C A$ is a cellular automaton system, $\mathrm{A}^{\mathrm{N}}$ 
represents cellular space, $\mathrm{N}$ represents the cellular space dimension; $\Sigma$ represents cellular state set; $\mathrm{E}$ represents the boundary type of cellular automata, the widely used are Von Neumann, Moore and extended Moore type; $\mathrm{F}$ is the state transition function, the next time cellular state transition function calculated by the current cellular status and its neighborhood cellular state.

Cellular automata model can show a very good local micro effect, but also has some shortcomings: each cell is changed under the influence of local area network, which can't provide a direct expression of the impact of complex macro factors in the network. Bass model is a kind of macroscopic model, the combination of Bass model and cellular automata model can overcome the lack of macro impact of cellular automata model, and better simulate the propagation law of public opinion in real network. Considering the views of netizens are affected by adjacent individual tendency and external macro influence, added the neighborhood influence degree, the external influence degree and the external influence on the basis of traditional model.

Set the network public opinion cell space as a $\mathrm{N} \times \mathrm{N}$ two-dimensional array, for the convenience of calculation, define the boundary conditions of cyberspace is cyclical, connect the upper and lower, left and right in the two-dimensional space. Each element corresponds to a netizen which is the tendency of a cell; cellular initial tendency has -1, 0,1 three kinds of finite discrete values, the range of the state transition is [-1, 1]. Every cell has a certain firm on their own point of view, firmness $K$ ranges $[0,1]$.Considering individual is affected by neighborhood tendency and external influence, define the cellular neighborhood influence degree $O$, value ranges [0,1]; Cellular external influence degree $P$, in the range of $[0,1]$, and $O+P=1$. Define the external influence $Q$, in the range of $[-1,1]$, the overall transmission is influenced by public opinion trends $Q w$ and news media and subjective factors, such as the news media and government guidance $Q e$, as shown in Equation (1)

$$
\mathrm{Q}=\mathrm{Q}_{\mathrm{w}}+\mathrm{Q}_{\mathrm{e}}
$$

Considering the guidance of government and news media usually appear in the event propagation process, so the initial $\mathrm{Qe}=0$, external influence initial value takes the overall trend of network public opinion Qw, its calculation method is shown as Equation (2)

$$
\mathrm{Q}_{\mathrm{w}}=\frac{1}{\mathrm{n} \times \mathrm{n}} \sum_{\mathrm{i}=1}^{\mathrm{n}} \sum_{\mathrm{j}=1}^{\mathrm{n}} \mathrm{S}_{\mathrm{i}, \mathrm{j}}(\mathrm{t})
$$

Cellular state transition rules considering external influence. When firmness $K=1$ in extreme cases, the tendency of cells is not affected by other cells. When $K \in[0,1)$, the conversion function $S_{i, j}(t+1)$ of cellular tendency in $\mathrm{t}+1$ is shown as Equation (3):

$$
\begin{aligned}
& S_{i, j}(t+1)=S_{i, j}(t) \times K+(1-K)\left\{\frac { 1 } { 8 } \left[S_{i-1, j-1}(t)+S_{i-1, j}(t)+S_{i, j+1}(t)+S_{i, j-1}(t)+\right.\right. \\
& \left.\left.S_{i, j+1}(t)+S_{i+1, j-1}(t)+S_{i+1, j}(t)+S_{i+1, j+1}(t)\right] \times 0+P \times Q\right\}
\end{aligned}
$$

Divide cell tendency degree by coarse grained and fine grained methods, the coarse grained method is to make statistical analysis according to $[-1,-\varepsilon),[-\varepsilon, \varepsilon]$ and $(\varepsilon, 1], \varepsilon$ is a minimum. The fine-grained classification is to make statistical analysis by dividing $[-1,1]$ into $n(n>3)$ internals according to the tendency.

Cell traversal algorithm is as follows. In this paper, neighborhood is fixed.

(1) Initialize parameter, set traversal order;

(2) Computing overall trend by Eq.2

(3) Calculate State by Eq.3;

(4) Repeat (2),3);

(5) Coarse grained trend analysis;

6) Fine grained trend analysis;

(7) Output.

Coarse-grained tendency degree algorithm. In order to study the overall tendency of cellular space, coarse grained analysis method is used. When the absolute value of $S_{i, j}(t)$ is very small, it is 
equal to 0 . The range of $\varepsilon$ in the Equation $(4)$ is $(0,1)$.

$$
\mathrm{S}_{\mathrm{i}, \mathrm{j}}(\mathrm{t}) \text { means }\left\{\begin{array}{c}
\text { opposed, }-1 \leq \mathrm{S}_{\mathrm{i}, \mathrm{j}}(\mathrm{t})<-\varepsilon \\
\text { neutral, }-\varepsilon \leq \mathrm{S}_{\mathrm{i}, \mathrm{j}}(\mathrm{t}) \leq \varepsilon \\
\text { supported, } \varepsilon<\mathrm{S}_{\mathrm{i}, \mathrm{j}}(\mathrm{t}) \leq 1
\end{array}\right.
$$

Fine-grained tendency degree algorithm. Count the cellular number in each subinterval by fine grained method, the interval [-1,1] is divided into 10 equal parts, as shown in Fig.1. Define a one-dimensional array of length $10, F[0 \ldots 9]$, when $S_{i, j}(t)$ falls on the $k$-th interval, the corresponding interval is $\mathrm{F}[\mathrm{k}-1]++$.

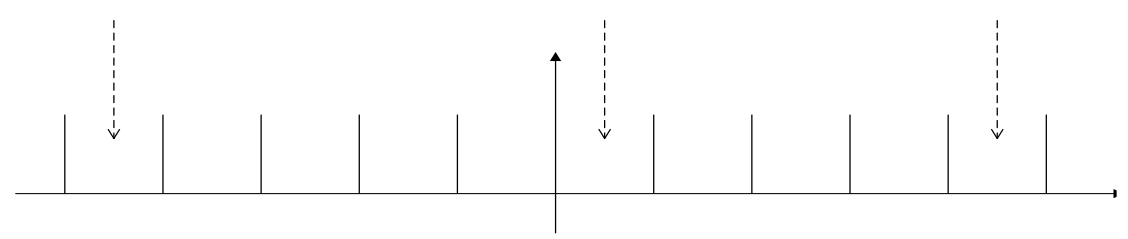

Fig.1 Interval [-1,1] 10 equal division

\section{The simulation experiment}

In this paper, Matlab2012b simulation software is used to simulate the network public opinion propagation based on Bass and cellular automata model, and the results are analyzed.

Initial conditions: Create a $21 \times 21$ cellular space; take $\varepsilon=0.15$ in the formula(4); the initial tendency of cells in accordance with supported tendency cell 205, neutral tendency cell 111, opposing tendency cell 125 distributes randomly. $25 \%$ cellular firmness is $0.75,10 \%$ cellular firmness is 0.5 , $65 \%$ cellular firmness is 0.25 , with random distribution.

Experiment 1: The tendency of cellular is affected by neighborhood cells, and is not affected by external overall trends.

Experiment 2: The neighborhood influence degree $O$, according to $65 \%$ of cells' neighborhood influence degree is $0.75,10 \%$ of cells' is $0.5,25 \%$ of cells' is 0.25 with random distribution. Correspondingly, the external influence degree $P=1-O$.

Experiment 3: Cellular neighborhood influence degree $O$, according to $25 \%$ of cells' neighborhood influence degree is $0.75,10 \%$ of cells' is $0.5,65 \%$ of cells' is 0.25 with random distribution. Correspondingly, the external influence degree $P=1-O$.

Experiment 4: The tendency of cell is affected by the external overall trend, and is not affected by neighborhood cells.

In experiment 1, 2, 3, 4, traverse all cells 8 times, get coarse-grained and fine-grained curve.

Simulation results and analysis

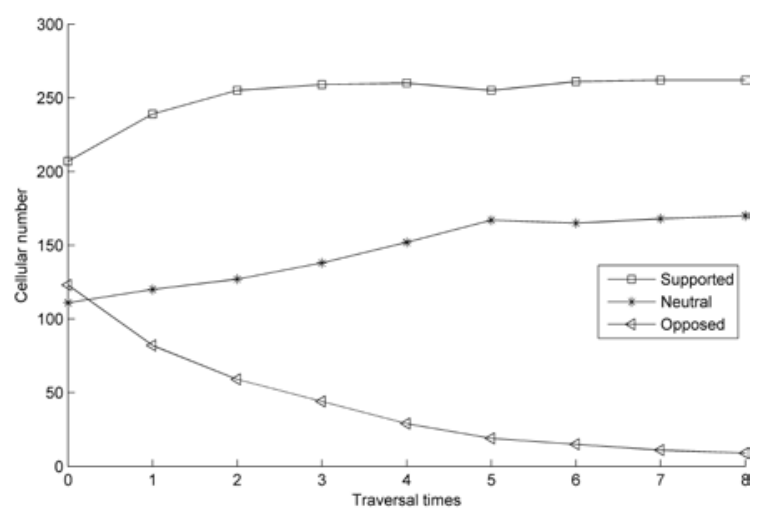

Experiment 1

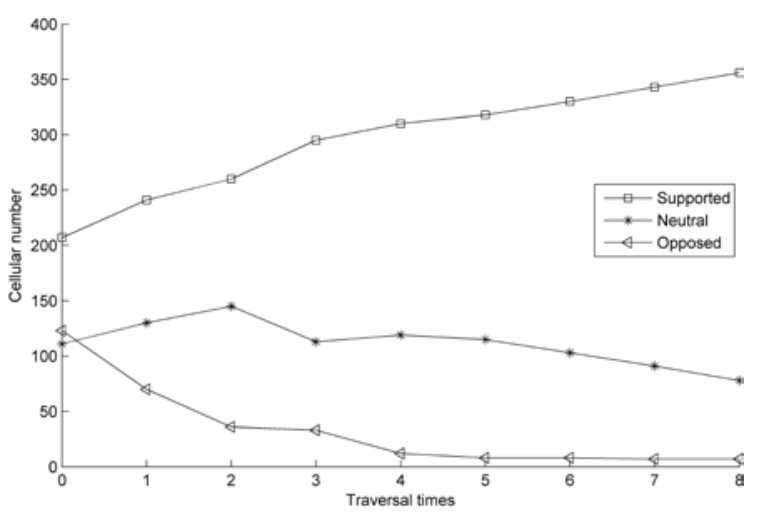

Experiment 2 


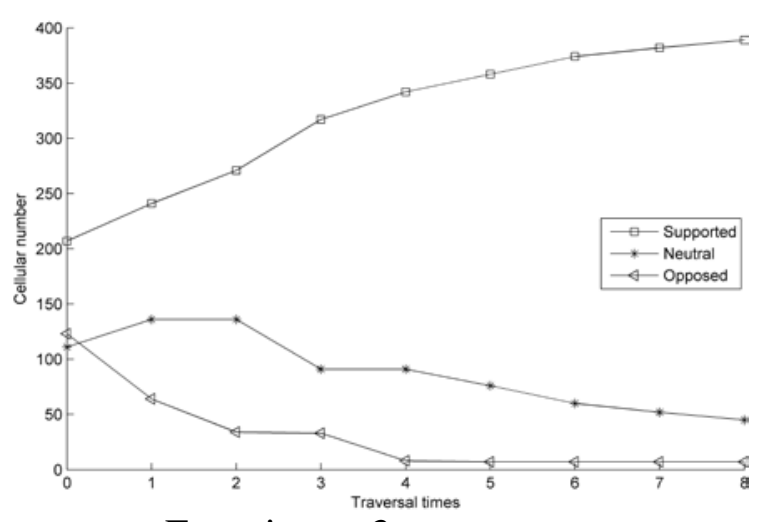

Experiment 3

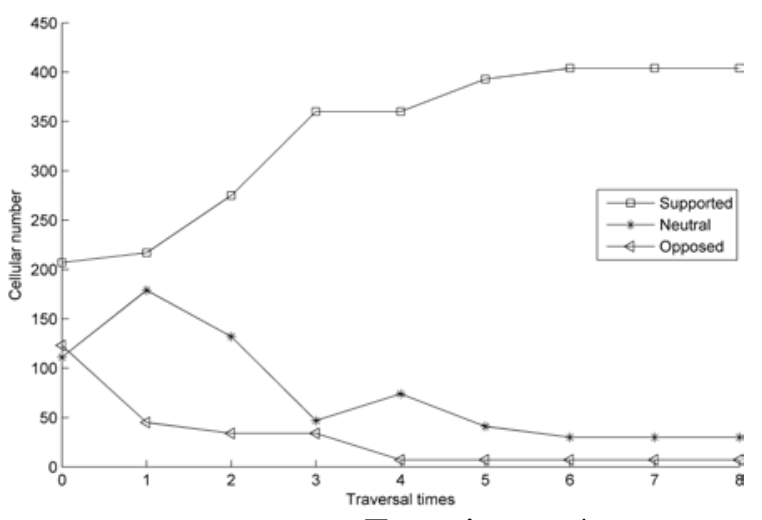

Experiment 4

Fig. 2 Coarse-grained curve

(1)The overall tendency distribution

As shown in Fig.2, the number of individuals holding "support" in the initial distribution is much more than the number of "opposition" and "neutral", after a certain period of time, the number of "support" individuals increased to the maximum, the number of "against" close 0 . In experiment 1 the number of "neutral" individuals increased about $50 \%$ after evolution without considering the external influence. Considering the external influence, "neutral" individual number decreased by about 30\% in experiment 2, decreased by about 55\% in experiment 3, decreased by about $70 \%$ in experiment 4 .

With the spread of public opinion, the number of "neutral" individuals will decrease with the increase of majority opinion in real life. Therefore, considering the condition of external influence is closer to the actual situation of public opinion propagation.

At the beginning the number of "support" grew rapidly, with the change of time, the growth trend gradually slowed down, finally leveled off, this is consistent with the dissemination of public opinion in the reality. The longer public opinion spread, the greater the impact on the entire network of public opinion, so the guidance of public opinion should be timely.

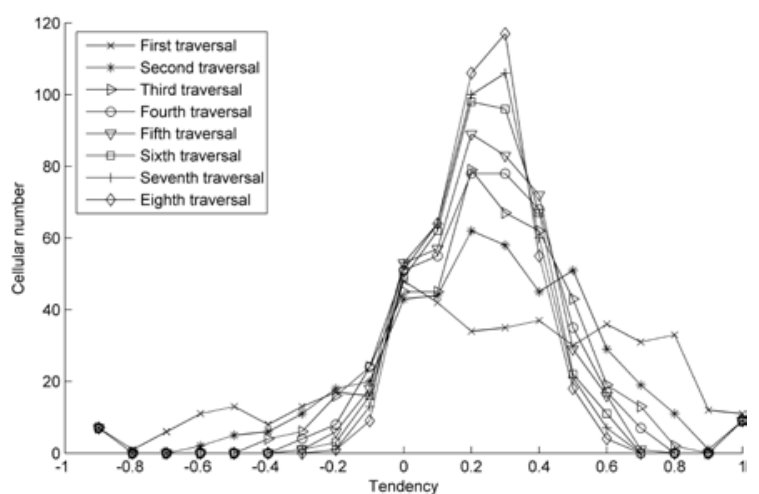

Experiment 1

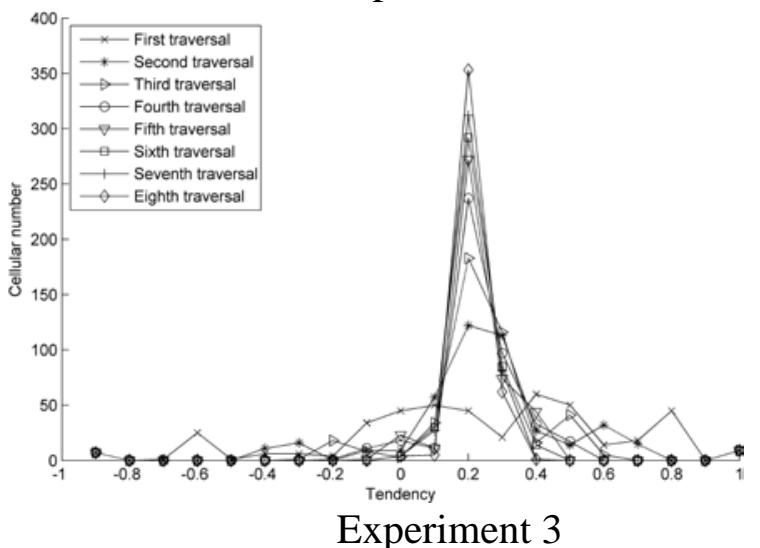

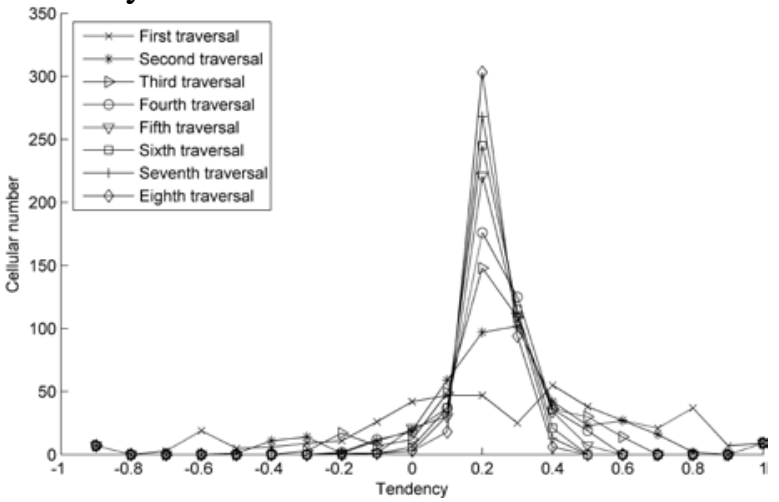

Experiment 2

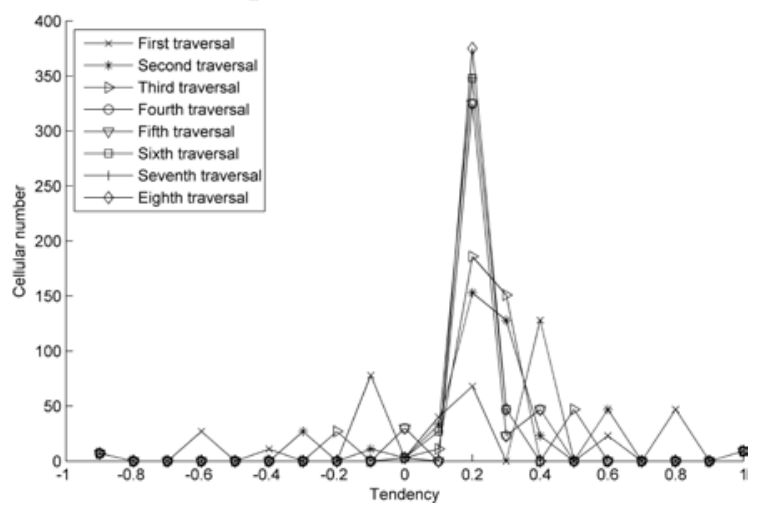

Experiment 4

Fig.3 Fine-grained curve 
(2)The influence of $\mathrm{O}$ and $\mathrm{P}$ values

In order to compare the degree of cell tendency, defined the homogenization as cells with the larger initial proportion accounts for the total cell number in the evolution process. Individual opinions are affected by both neighborhood individual and external influence in real opinion propagation. In Fig.2, the initial tendency and firmness of cellular were fixed, cellular homogenization rate increased by about $30 \%$ in experiment 2 , $40 \%$ in experiment 3 . Therefore, the smaller individual is affected by neighborhood influence degree $\mathrm{O}$ and the bigger the external influence degree $\mathrm{P}$, the higher homogenization when public opinion achieves stable.

(3)The analysis of fine grained tendency curve

In Fig.3, the initial tendency of individual shows a multivariate and scatter distribution, it's consistent with the real network. In experiment 1, 2, 3, 4, like-minded individuals began to gather, formed a gathering area after a period of time, the tendency of individuals converged in the core area of has a high aggregation degree. Considering the conditions of external influence, as can be seen from the fine-grained curve of experiment 2 and 3, after a certain number of iterations, the group's view will normalized, the number of large tendency individuals is closed to 0 , the group tendency gathered in a relatively eclectic area.

In the evolution of network public opinion, opinions of like-minded netizens gathered together with the change of time, netizens with extreme views gradually formed a relatively compromise views which are closed to the majority of people after a group communication and discussion, group opinion achieved a consistent trend at last.

Experiment 5: Initial state is the same as experiment 2, observed a rapid increase in the number of the "support" individuals in the previous two iterations, introduced $Q e=-0.1$ and $Q e=-0.2$ two negative external influence flows in the third iteration of simulation experiment 2 , the evolution trend of public opinion is shown in Fig.4.
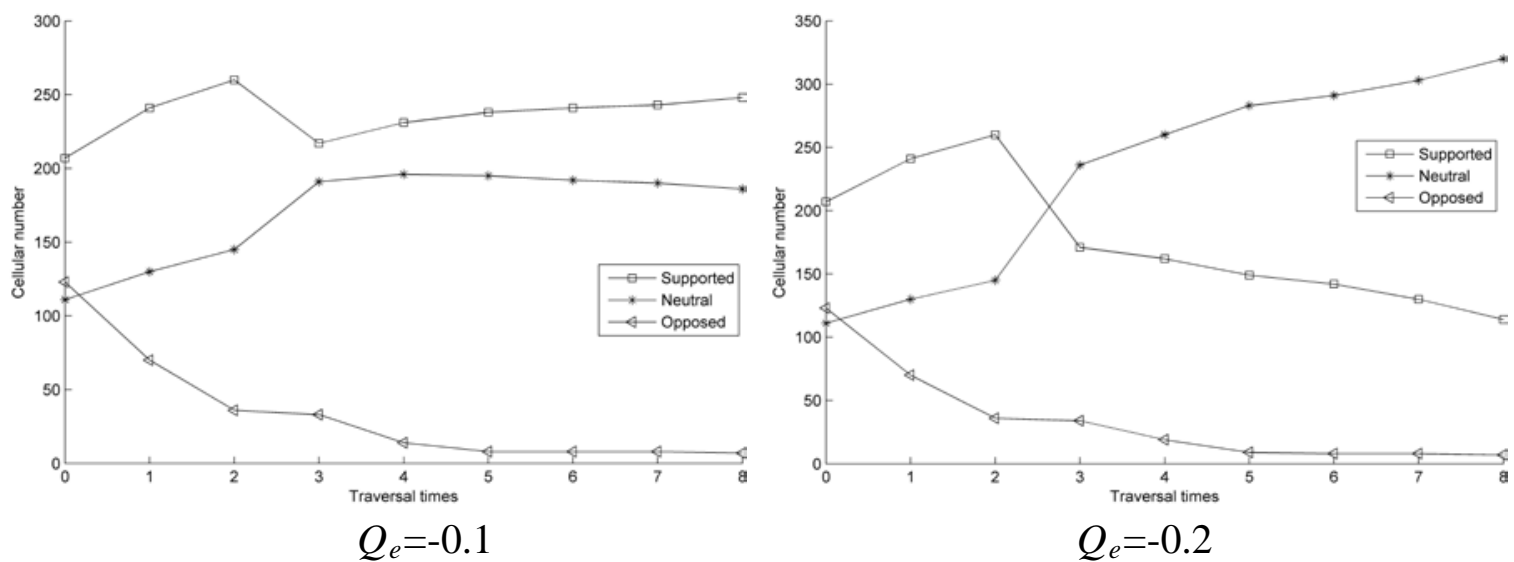

Fig.4 Introduce external influence flow

(4)Introduce the external influence flow $Q e$

In Fig.2, the public opinion grew rapidly at beginning, introduced two negative external influence flows in the third iteration. As can be seen from Fig.4, after introducing a negative influence flow $Q e=-0.1$, the spread of public opinion got contained quickly, public opinion quickly stabilized. After introducing the negative influence flow $Q e=-0.2$, public opinion occurred in reverse, "neutral" opinions accounted for the majority. Experimental results showed that external influence has a significant impact on the propagation process and evolution of public opinion.

\section{Conclusion}

Considering Internet users' views are influenced by the people around them and the overall trend of public opinion as well as the external macro factors such as the government and the news media. On the basis of Bass theory, introduced neighborhood influence degree, external influence degree and 
external influence, proposed a hybrid model for the evolution of online public sentiment. Draw the following conclusions:

(1)Considering the conditions of external influence, groups tendency rapidly gather to the majority opinion, "against" opinions were quickly assimilated by the majority opinion, the number of "neutral" individuals rapidly reduced to about $10 \% \sim 18 \%$ of the total, the overall tendency of public opinion shows a high degree of convergence.

(2)The value of neighborhood influence degree $\mathrm{O}$ and external influence $\mathrm{P}$ will affect the number of individuals with different views after reaching stability, the smaller neighborhood influence degree $\mathrm{O}$ and the bigger the external influence degree $\mathrm{P}$ is, the higher homogenization when public opinion reaches a stable state.

(3)After a period of time, group views will be normalized, not in extreme favor or opposition, but close to the majority opinion, formed a relatively eclectic core opinion gathering area.

The hybrid model in this paper reveals some laws of network public opinion, which is helpful to analyze, predict, manage and control the spread of public opinion. The study also has some shortcomings, there is no distinction between general netizens and important netizens, did not consider the influence sphere of netizens is different. These factors are the focus of the next study.

\section{Acknowledgement}

In this paper, the research was sponsored by Supported by Program for Innovative Research Team (in Science and Technology) in University of Henan Province (Project No.17IRTSTHN010), Technological Innovation Team of Henan University of Science and Technology (Project No.2015XTD011) and Major Cooperative Engagement Fund of Henan University of Science and Technology (Project No.2015ZDCXY03), Research on science and technology project of Henan Province (Project No. 152102210277), Project of industry university research cooperation in Henan Province (Project No. 152107000027).

\section{References}

[1] Alves S G. Electoral surveys influence on the voting processes: a cellular automata model. Physica A, 316(1/4):601- 614, 2002.

[2] Zeng Xiankui. Study of the Cellular Automaton Models of Public Opinion Based on Majority Rule and Cooperation Rule, Guangxi Normal University, 2007.

[3] Fang Wei, He Liujin, Sun Kai, Zhao Peng, Study on dissemination model of network public sentiment based on cellular automata. Journal of Computer Applications, 03:751-755, 2010.

[4] Liu Muren, Deng Minyi, Kong Lingjiang, A Model For the Evolution of public Opinion Based on Cellular Automata[J]. Journal of Guangxi Normal University, 02:1-4, 2002.

[5] Zeng Xiangping, Fang Yong, Yuan Yuan et al, Motivation model for online public opinion based on cellular automata. Journal of Computer Applications, 11:2686-2688+ 2714, 2007.

[6] Wang Gensheng, Le Zhongjian, Lu Xu et al, A Model For the Evolution of Public Opinion in the Web Based on Migration Cellular Automata. Journal of the China Society for Scientific and Technical Information, 30(6):570-576, 2011. 